\title{
ABSTRACT OF COMPUTER PROGRAMS
}

UWBIC

Language

Author

System

Abstract

Documentation
University of Washington BASIC Interpretive Compiler FORTRAN IV

William F. Sharpe, Professor of Finance, Business Economics and Quantitative Methods Graduate School of Business Administration University of Washington

UWBIC can be used with little or no alteration on virtually any medium-to-large-scale computer provided with a FORTRAN IV compiler and therefore is relatively machine independent. Thus far, it has been run on the IBM 7044, IBM 7094, Univac 1108 and $\mathrm{CDC} 6600$.

UWBIC consists of eight subroutines. It accepts, as inputs, programs written in the BASIC language and data to be operated on by such programs. The output produced will be either (1) diagnostic messages indicating errors in the program written in BASIC or (2) output produced by following the instructions written in BASIC with data provided. The average time needed to compile and execute jobs prepared by beginning students is about 10 seconds on the 7094 (after loading the system).

a) Reference manual and program listing: University of Washington BASIC Interpretive Compiler, Technical Report No. 3. (W. F. Sharpe), $\$ 2.00$. Order from:

Director of Publications

Graduate School of Business Administration University of Washington

Seattle, Washington 98105

b) Program and sample data (card or tape copy) available for $\$ 20.00$ from:

Computer Center Software Management and Information

University of Georgia

Athens, Georg1a 30601

c) Student Manual of the BASIC language, BASIC: An Introduction to Computer Programming Using the BASIC Language, W. F. Sharpe, 1967 New York, The Free Press (A division of Macmillan). \$3.95. 